K. Shiohama and H. Xu

Nagoya Math. J.

Vol. 150 (1998), 105-134

\title{
A GENERAL RIGIDITY THEOREM FOR COMPLETE SUBMANIFOLDS
}

\author{
KATSUHIRO SHIOHAMA AND HONGWEI XU
}

\begin{abstract}
Making use of 1-forms and geometric inequalities we prove the rigidity property of complete submanifolds $M^{n}$ with parallel mean curvature normal in a complete and simply connected Riemannian $(n+p)$-manifold $N^{n+p}$ with positive sectional curvature. For given integers $n, p$ and for a nonnegative constant $H$ we find a positive number $\tau(n, p) \in(0,1)$ with the property that if the sectional curvature of $N$ is pinched in $[\tau(n, p), 1]$, and if the squared norm of the second fundamental form is in a certain interval, then $N^{n+p}$ is isometric to the standard unit $(n+p)$-sphere. As a consequence, such an $M$ is congruent to one of the five models as seen in our Main Theorem.
\end{abstract}

\section{$\S 0$. Introduction}

An important problem in differential geometry is the study of relations between the geometric structure and the geometric invariants of Riemannian submanifolds. After the pioneering work of Simons $[\mathrm{S}]$ the following result, known as the rigidity theorem for submanifolds containing minimal cases, was proved first by Lawson [L1], Chern-do Carmo-Kobayashi [CDK] and later $\mathrm{Li}$ and $\mathrm{Li}[\mathrm{LL}]$ and finally by the second author $[\mathrm{X} 1]$, as stated

THEOREM 0.1. For given constant $H \geq 0$ and positive integers $n(\geq$ $2), p$ there exists a positive number $C(n, p, H)$ with the following property: If $M^{n}$ is a closed submanifold in the standard unit $(n+p)$-sphere $\mathbf{S}^{n+p}(1)$ with parallel mean curvature normal field having norm $H$, and if $S$ is the squared norm of the second fundamental form satisfying

$$
S \leq C(n, p, H),
$$

then $M$ is congruent to one of the following:

(1) $\mathbf{S}^{n}\left(\frac{1}{\sqrt{1+H^{2}}}\right)$

Received August 22, 1996.

Research supported in part by Grant-in-Aid for Co-operative Research No. 06302007 from the Ministry of Education, Science and Culture.

The second author was supported by the NNSFC. Grant No. 1957107 (China). 
(2) the isoparametric hypersurface $\mathbf{S}^{n-1}\left(\frac{1}{\sqrt{1+\lambda^{2}}}\right) \times \mathbf{S}^{1}\left(\frac{\lambda}{\sqrt{1+\lambda^{2}}}\right)$ in $\mathbf{S}^{n+1}(1)$,

(3) one of the Clifford minimal hypersurfaces $\mathbf{S}^{k}\left(\sqrt{\frac{k}{n}}\right) \times \mathbf{S}^{n-k}\left(\sqrt{\frac{n-k}{n}}\right)$ in $\mathbf{S}^{n+1}(1)$, for $k=1, \cdots, n-1$,

(4) the Clifford torus $\mathbf{S}^{1}\left(r_{1}\right) \times \mathbf{S}^{1}\left(r_{2}\right)$ in $\mathbf{S}^{3}(r)$ with constant mean curvature $H_{0}$, where $r_{1}, r_{2}=\left[2\left(1+H^{2}\right) \pm 2 H_{0}\left(1+H^{2}\right)^{1 / 2}\right]^{-1 / 2}, r=$ $\left(1+H^{2}-H_{0}^{2}\right)^{-1 / 2}$, and $0 \leq H_{0} \leq H$,

(5) the Veronese surface in $\mathbf{S}^{4}\left(\frac{1}{\sqrt{1+H^{2}}}\right)$.

Here $\lambda$ and $C(n, p, H)$ are given by

$$
\lambda=\frac{1}{2(n-1)}\left[n H+\sqrt{n^{2} H^{2}+4(n-1)}\right]
$$

and

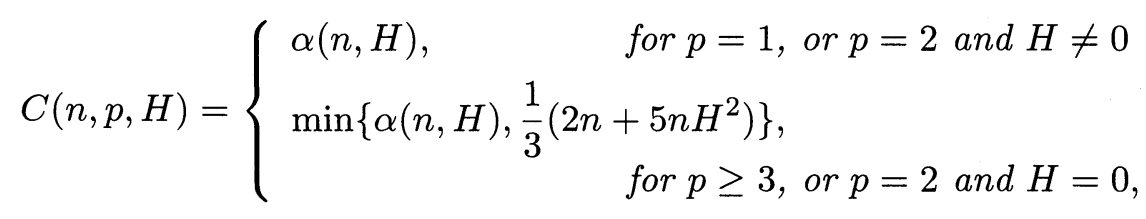

where

$$
\alpha(n, H)=n+\frac{n^{3}}{2(n-1)} H^{2}-\frac{n(n-2)}{2(n-1)} \sqrt{n^{2} H^{4}+4(n-1) H^{2}} .
$$

Note that the special case where $p=1$ and $H \neq 0$ was proved independently by Alencar and do Carmo in [AdC]. Also note that Theorem 0.1 was obtained under the assumption that the ambient space is the round sphere. The existence of parallel mean curvature normal field imposes very nice properties to submanifolds, whatever the ambient spaces are. In fact the second author proved in [X2] the rigidity for compact minimal submanifolds in pinched Riemannian manifolds, as stated

THEOREM 0.2. ([X2]) For given positive integers $n \geq 2$, $p$ there exists a number $\delta(n, p)$ with $0<\delta(n, p)<1$ with the following properties: If $M^{n}$ is an oriented closed minimal submanifold in a complete simply connected manifold $N^{n+p}$ whose sectional curvature $K_{N}$ satisfies $\delta(n, p) \leq K_{N} \leq 1$ and if

$$
\beta(n, p)(1-c) \leq S \leq n-\frac{n}{3} \operatorname{sgn}(p-1)-\gamma(n, p)(1-c)
$$


where $c:=\inf K_{N}$, then $N$ is isometric to $\mathbf{S}^{n+p}(1)$. Moreover $M$ is congruent to one of the following:

(1) $\mathbf{S}^{n}(1)$,

(2) the Clifford minimal hypersurfaces $\mathbf{S}^{k}\left(\sqrt{\frac{k}{n}}\right) \times \mathbf{S}^{n-k}\left(\sqrt{\frac{n-k}{n}}\right)$ in $\mathbf{S}^{n+1}(1)$, for $k=1, \cdots, n-1$,

(3) the Veronese surface in $\mathbf{S}^{4}(1)$.

Here $\beta(n, p), \gamma(n, p)$ and $\delta(n, p)$ are given as

$$
\begin{aligned}
\beta(n, p):= & \frac{1}{12}[p n(n-1)(52 n-50)]^{1 / 2} \\
\gamma(n, p):= & n+\frac{2}{3}(p-1)(n-1)^{1 / 2}+\frac{1}{12}[p n(n-1)(52 n-50)]^{1 / 2} \\
\delta(n, p):= & 1-n(3-\operatorname{sgn}(p-1))\left\{3 n+2(p-1)(n-1)^{1 / 2}\right. \\
& \left.+\frac{\sqrt{2}}{2}[p n(n-1)(26 n-25)]^{1 / 2}\right\}^{-1}
\end{aligned}
$$

The purpose of this paper is to relax the special closed submanifolds in the above results to complete submanifolds with parallel mean curvature normal fields, and the ambient space to general Riemannian manifold $N^{n+p}$. Thus we obtain the generalization of Theorems 0.1 and 0.2 .

MAIN TheOREM. For given positive integers $n(\geq 2), p$ and a nonnegative constant $H$ there exists a number $\tau(n, p)$ such that $0<\tau(n, p)<1$ with the following properties: If $M^{n}$ is an oriented complete submanifold with parallel mean curvature normal field with its norm $H$ in a complete and simply connected $(n+p)$-dimensional Riemannian manifold with $\tau(n, p) \leq$ $K_{N} \leq 1$, and if

$$
\begin{aligned}
& n H^{2}+A_{1}(n, p)(1-c)+A_{2}(n, p)\left[\left(1+H^{2}\right) H\right]^{1 / 2}(1-c)^{1 / 4} \\
& \quad \leq S \leq C(n, p, H)-B_{1}(n, p)(1-c)-B_{2}(n, p)\left[\left(1+H^{2}\right) H\right]^{1 / 2}(1-c)^{1 / 4}
\end{aligned}
$$

where $c:=\inf K_{N}$, then $N$ is isometric to $\mathbf{S}^{n+p}(1)$. Moreover,

1. If $\sup _{M} S<\alpha(n, H)$, then $M$ is congruent to either $\mathbf{S}^{n}\left(\frac{1}{\sqrt{1+H^{2}}}\right)$ or the Veronese surface in $\mathbf{S}^{4}\left(\frac{1}{\sqrt{1+H^{2}}}\right)$.

2. If $M$ is compact, then $M$ is congruent to one of the following: 
(1) $\mathbf{S}^{n}\left(\frac{1}{\sqrt{1+H^{2}}}\right)$,

(2) the isoparametric hypersurface $\mathbf{S}^{n-1}\left(\frac{1}{\sqrt{1+\lambda^{2}}}\right) \times \mathbf{S}^{1}\left(\frac{\lambda}{\sqrt{1+\lambda^{2}}}\right)$ in $\mathbf{S}^{n+1}(1)$,

(3) one of the Clifford minimal hypersurfaces $\mathbf{S}^{k}\left(\sqrt{\frac{k}{n}}\right) \times \mathbf{S}^{n-k}\left(\sqrt{\frac{n-k}{n}}\right)$ in $\mathbf{S}^{n+1}(1)$, for $k=1, \cdots, n-1$,

(4) the Clifford torus $\mathbf{S}^{1}\left(r_{1}\right) \times \mathbf{S}^{1}\left(r_{2}\right)$ in $\mathbf{S}^{3}(r)$ with constant mean curvature $H_{0}$, where $r_{1}, r_{2}=\left[2\left(1+H^{2}\right) \pm 2 H_{0}\left(1+H^{2}\right)^{1 / 2}\right]^{-1 / 2}, r=$ $\left(1+H^{2}-H_{0}^{2}\right)^{-1 / 2}$, and $0 \leq H_{0} \leq H$,

(5) the Veronese surface in $\mathbf{S}^{4}\left(\frac{1}{\sqrt{1+H^{2}}}\right)$.

Here $C(n, p, H), \alpha(n, H)$ and $\lambda$ are defined as in Theorem 0.1 .

The constants $\tau(n, p), A_{1}(n, p), A_{2}(n, p), B_{1}(n, p)$ and $B_{2}(n, p)$ are precisely given in the proof (see $\S 3$ ). In the case where $M$ is not oriented, we can obtain similar results by using the Riemannian double cover. Our proof method is quite different from the previous ones developed in Theorems 0.1 and 0.2. In contrast to the proofs of Theorems 0.1 and 0.2 , our proof does not require the generalized maximum principle and the generalized Simons inequality, but the use of two distinct differential 1-forms and integral inequalities for the semi-norms of the second fundamental form of $M$. The crucial point of our proof is to verify that $c=1$. The closed minimal case in our Main Theorem has already been established in Theorem 0.2 , and hence $H \neq 0$ is assumed throughout $\S 2$ and $\S 3$. In due course of the proof of our Main Theorem we obtain geometric inequalities (see Theorems 2.4, 2.8 , etc.) by which the rigidity results for compact cases are obtained (see Theorems 3.1, 3.3 and 3.4). In complete case we show by computations that the Ricci curvature is bounded below by a positive constant, and hence it reduces to compact case.

The paper is organized as follows. Local formulas and propositions are prepared in $\S 1$. In $\S 2$ we present two geometric inequalities for the seminorms of the second fundamental form. In $\S 3$ we investigate the rigidity of closed submanifolds with parallel mean curvature normal field in a pinched manifold. In $\S 4$ we discuss complete submanifold with parallel mean curvature normal field and complete the proof of our Main Theorem.

The second author would like to express his thanks to Professor P. $\mathrm{Li}$ for his valuable suggestions. 


\section{$\S 1$. Preliminaries}

Throughout this paper let $M^{n}$ be an $n$-dimensional connected and complete Riemannian manifold isometrically immersed into an $(n+p)$ dimensional Riemannian manifold $N^{n+p}$. The following convention of indices are used throughout.

$1 \leq A, B, C, \cdots \leq n+p, \quad 1 \leq i, j, k, \cdots \leq n, \quad n+1 \leq \alpha, \beta, \gamma, \cdots \leq n+p$.

Choose an orthonormal frame field $\left\{e_{A}\right\}$ in a neighborhood of a point $p \in M$ such that the $e_{i}$ 's span the tangent space $T_{p} M$ to $M$ at $p$. Let $\left\{\omega_{A}\right\}$ be the dual frame fields of $\left\{e_{A}\right\}$ and $\left\{\omega_{A B}\right\}$ the connection 1-forms of $N$. Restricting these forms to $M$, we have

$$
\omega_{\alpha i}=\sum_{j} h_{i j}^{\alpha} \omega_{j}, \quad h_{i j}^{\alpha}=h_{j i}^{\alpha} .
$$

Let $a(x), b(x)$ for $x \in N$ be the minimum and maximum of $K_{N}$ at that point and $c, d$ the infimum and supremum of $a, b: N \rightarrow \mathbf{R}$ over $N$. The curvature tensors of $N, M$ are denoted by $K_{A B C D}, R_{i j k l}$, and the normal curvature tensor of $M$ by $R_{\alpha \beta k l}$ respectively. Let $R$ be the scalar curvature of $M$. The second fundamental form of $M$ is denoted by $h$ and the mean curvature normal field by $\xi$. Set $H:=\|\xi\|$, the mean curvature of $M$ and $S$ the squared norm of $h$. We then have

$$
\begin{aligned}
h & =\sum_{\alpha, i, j} h_{i j}^{\alpha} \omega_{i} \otimes \omega_{j} \otimes e_{\alpha}, \\
\xi & =\frac{1}{n} \sum_{\alpha, i} h_{i i}^{\alpha} e_{\alpha}, \\
R_{i j k l} & =K_{i j k l}+\sum_{\alpha}\left(h_{i k}^{\alpha} h_{j l}^{\alpha}-h_{i l}^{\alpha} h_{j k}^{\alpha}\right), \\
R_{\alpha \beta k l} & =K_{\alpha \beta k l}+\sum_{i}\left(h_{i k}^{\alpha} h_{i l}^{\beta}-h_{i l}^{\alpha} h_{i k}^{\beta}\right) .
\end{aligned}
$$

The scalar curvature $R$ of $M$ is given by

$$
R=\sum_{i, j} K_{i j i j}+n^{2} H^{2}-S
$$

Definition 1.1. We say that $M$ admits parallel mean curvature normal field iff $\xi$ is parallel in the normal bundle over $M$. 
We assume that $M$ admits a parallel mean curvature normal field and that $H \neq 0$. Choose $e_{n+1}$ such that it is parallel to $\xi$ and $\operatorname{tr} H_{n+1}:=\operatorname{tr}\left(h_{i j}^{n+1}\right)=$ $n H$. Then, setting $H_{\alpha}$ the $(n \times n)$-matrix with $(i, j)$ component $h_{i j}^{\alpha}$, we observe that $\operatorname{tr} H_{\beta}=0$ for all $\beta \geq n+2$. The squared norm $S$ of the second fundamental form is divided into two parts as follows.

$$
S_{H}:=\operatorname{tr} H_{n+1}^{2}, \quad S_{I}:=\sum_{\beta \neq n+1} \operatorname{tr} H_{\beta}^{2} .
$$

The following proposition is immediate from the definition, and the proof is omitted here.

Proposition 1.2. If $M$ admits parallel mean curvature normal field $\xi$, then either $H \equiv 0$ or $H$ is non-zero constant and $H_{\alpha} H_{n+1}=H_{n+1} H_{\alpha}+$ $\left(K_{n+1 \alpha i j}\right)_{n \times n}$.

Denoting the covariant derivatives of $h_{i j}^{\alpha}$ by $h_{i j k}^{\alpha}$ and $h_{i j k l}^{\alpha}$ respectively, we have

$$
\begin{aligned}
& \sum_{k} h_{i j k}^{\alpha} \omega_{k}=d h_{i j}^{\alpha}+\sum_{s} h_{s j}^{\alpha} \omega_{i s}+\sum_{s} h_{i s}^{\alpha} \omega_{j s}+\sum_{\beta} h_{i j}^{\beta} \omega_{\alpha \beta} \\
& \sum_{l} h_{i j k l}^{\alpha} \omega_{l}=d h_{i j k}^{\alpha}+\sum_{s} h_{s j k}^{\alpha} \omega_{i s}+\sum_{s} h_{i s k}^{\alpha} \omega_{j s}+\sum_{s} h_{i j s}^{\alpha} \omega_{k s}+\sum_{\beta} h_{i j k}^{\beta} \omega_{\alpha \beta} .
\end{aligned}
$$

We then have

$$
h_{i j k}^{\alpha}-h_{i k j}^{\alpha}=-K_{\alpha i j k}
$$

and the Ricci formula

$$
h_{i j k l}^{\alpha}-h_{i j l k}^{\alpha}=\sum_{s} h_{s j}^{\alpha} R_{s i k l}+\sum_{s} h_{i s}^{\alpha} R_{s j k l}+\sum_{\beta} h_{i j}^{\beta} R_{\beta \alpha k l} .
$$

Let $K_{\alpha i j k l}$ be the covariant derivative of $K_{\alpha i j k}$ as the section of $T^{\perp}(M) \otimes$ $T^{*}(M) \otimes T^{*}(M) \otimes T^{*}(M)$ and $K_{A B C D ; E}$ the covariant derivative of $K_{A B C D}$ as the curvature tensor of $N$. Restricted to $M$ we have

$$
\begin{aligned}
\sum_{l} K_{\alpha i j k l} \omega_{l} & =d K_{\alpha i j k}+\sum_{s} K_{\alpha s j k} \omega_{i s} \\
+ & \sum_{s} K_{\alpha i s k} \omega_{j s}+\sum_{s} K_{\alpha i j s} \omega_{k s}+\sum_{\beta} K_{\beta i j k} \omega_{\alpha \beta}
\end{aligned}
$$


and

$$
\begin{aligned}
K_{\alpha i j k ; l}=K_{\alpha i j k l} & -\sum_{\beta} K_{\alpha \beta j k} h_{i l}^{\beta} \\
& -\sum_{\beta} K_{\alpha i \beta k} h_{j l}^{\beta}-\sum_{\beta} K_{\alpha i j \beta} h_{k l}^{\beta}+\sum_{m} K_{m i j k} h_{m l}^{\alpha} .
\end{aligned}
$$

DEFINITION 1.3. $\quad M$ is called a submanifold with parallel second fundamental form iff $h_{i j k}^{\alpha} \equiv 0$ for all $i, j, k$ and $\alpha . N$ is by definition a locally symmetric space iff $K_{A B C D ; E} \equiv 0$ for all $A, B, C, D, E$.

The Laplacian $\Delta h_{i j}^{\alpha}$ of the second fundamental form $h$ is defined by $\Delta h_{i j}^{\alpha}:=$ $\sum_{k} h_{i j k k}^{\alpha}$. We set $\nabla_{k} h_{i j}^{\alpha}:=h_{i j k}^{\alpha}$. For an $(n \times n)$-matrix $A=\left(a_{i j}\right)$ we denote by $N(A)$ the squared norm of $A$, i.e., $N(A):=\operatorname{tr}\left(A^{t} A\right)=\sum_{i, j} a_{i j}^{2}$. Then $N(A)=N\left(T A^{t} T\right)$ holds for every orthogonal $(n \times n)$-matrix $T$.

Proposition 1.4. (see [CDK], [LL]) For symmetric matrices $A_{n+1}$, $\cdots, A_{n+p}$ let $S_{\alpha \beta}:=\operatorname{tr}\left(A_{\alpha} A_{\beta}\right), S_{\alpha}:=S_{\alpha \alpha}=N\left(A_{\alpha}\right)$ and $S:=\sum_{\alpha} S_{\alpha}$. Then

$$
\sum_{\alpha, \beta} N\left(A_{\alpha} A_{\beta}-A_{\beta} A_{\alpha}\right)+\sum_{\alpha, \beta} S_{\alpha \beta}^{2} \leq\left(1+\frac{1}{2} \operatorname{sgn}(p-1)\right) S^{2}
$$

where $\operatorname{sgn}(\cdot)$ is the standard sign function. Moreover, equality holds if and only if at most two matrices $A_{\alpha}$ and $A_{\beta}$ are non-zero and they can be transformed simultaneously by an orthogonal matrix into scalar multiples of $\tilde{A}_{\alpha}$ and $\tilde{A}_{\beta}$ respectively where

$$
\tilde{A}_{\alpha}=\left(\begin{array}{ccccc}
0 & 1 & 0 & \cdots & 0 \\
1 & 0 & 0 & \cdots & 0 \\
0 & 0 & 0 & \cdots & 0 \\
\vdots & \vdots & \vdots & \ddots & \vdots \\
0 & 0 & 0 & \cdots & 0
\end{array}\right), \quad \tilde{A}_{\beta}=\left(\begin{array}{ccccc}
1 & 0 & 0 & \cdots & 0 \\
0 & -1 & 0 & \cdots & 0 \\
0 & 0 & 0 & \cdots & 0 \\
\vdots & \vdots & \vdots & \ddots & \vdots \\
0 & 0 & 0 & \cdots & 0
\end{array}\right)
$$

The following Proposition is seen in [G]. The proof is omitted here.

Proposition 1.5. Let $N$ be an $(n+p)$-dimensional Riemannian manifold. If $a \leq K_{N} \leq b$ is satisfied at a point, then the following estimates hold at that point.

(1) $\left|K_{A C B C}\right| \leq \frac{1}{2}(b-a)$, for $A \neq B$, 
(2) $\left|K_{A B C D}\right| \leq \frac{2}{3}(b-a)$, for all distinct indices $A, B, C, D$.

Proposition 1.6. Let $a_{1}, \cdots, a_{n}$ and $b_{1}, \cdots, b_{n}$ be real numbers satisfying $\sum_{i} a_{i}=\sum_{i} b_{i}=0, \sum_{i} a_{i}^{2}=a$ and $\sum_{i} b_{i}^{2}=b$. Then

$$
\left|\sum_{i} a_{i} b_{i}^{2}\right| \leq(n-2)[n(n-1)]^{-1 / 2} a^{1 / 2} b,
$$

where equality holds if and only if either $a b=0$, or at least $n-1$ pairs of numbers of $\left(a_{i}, b_{i}\right)$ 's are the same.

Proof. We only need to check the case where $n \geq 3$ and $a=b=1$. Consider the function

$$
f\left(x_{1}, \cdots, x_{n}, y_{1}, \cdots, y_{n}\right)=\sum_{i} x_{i} y_{i}^{2}
$$

subject to the constraint conditions

$$
\sum_{i} x_{i}=\sum_{i} y_{i}=0, \quad \sum_{i} x_{i}^{2}=\sum_{i} y_{i}^{2}=1 .
$$

The Lagrange multiplier is employed for the proof by setting

$$
\begin{aligned}
& F\left(x_{1}, \cdots, x_{n}, y_{1}, \cdots, y_{n}, \lambda, \mu, \nu, \sigma\right) \\
& =\sum_{i} x_{i} y_{i}^{2}+\lambda\left(\sum_{i} x_{i}^{2}-1\right)+\mu\left(\sum_{i} y_{i}^{2}-1\right)+\nu \sum_{i} x_{i}+\sigma \sum_{i} y_{i} .
\end{aligned}
$$

If $\left(x_{1}, \cdots, x_{n}, y_{1}, \cdots, y_{n}\right)$ is a critical point of $f$ with the critical value $T_{0}$ under the constraint conditions (1.11), we then have

$$
\begin{array}{r}
y_{i}^{2}+2 \lambda x_{i}+\nu=0, \\
2 x_{i} y_{i}+2 \mu y_{i}+\sigma=0 .
\end{array}
$$

From (1.11) and the above relations,

$$
\nu=-\frac{1}{n}, \quad \lambda=\frac{1}{2} \mu=-\frac{1}{2} \sum_{i} x_{i} y_{i}^{2}=-\frac{1}{2} T_{0} .
$$

Combining (1.12) and (1.14),

$$
T_{0}^{2}=-2 \lambda \sum_{i} x_{i} y_{i}^{2}=\sum_{i} y_{i}^{4}-\frac{1}{n} .
$$


Now we compute the maximum of the function

$$
g\left(z_{1}, \cdots, z_{n}\right):=\sum_{i} z_{i}^{4}-\frac{1}{n}
$$

under the constraint conditions

$$
\sum_{i} z_{i}=0, \quad \sum_{i} z_{i}^{2}=1
$$

From (1.16) we get

$$
\sum_{1 \leq i<j \leq n-1} z_{i} z_{j}=z_{n}^{2}-\frac{1}{2}
$$

Making use of (1.16), (1.17) and the Schwarz inequality,

$$
\begin{aligned}
g\left(z_{1}, \cdots, z_{n}\right) \\
\quad=\left(\sum_{i=1}^{n-1} z_{i}^{2}\right)^{2}-2 \sum_{1 \leq i<j \leq n-1} z_{i}^{2} z_{j}^{2}+z_{n}^{4}-\frac{1}{n} \\
\quad \leq\left(1-z_{n}^{2}\right)^{2}-\frac{4}{(n-1)(n-2)}\left(\sum_{1 \leq i<j \leq n-1} z_{i} z_{j}\right)^{2}+z_{n}^{4}-\frac{1}{n} \\
\quad=\frac{2 n(n-3)}{(n-1)(n-2)} z_{n}^{4}-\frac{2 n(n-3)}{(n-1)(n-2)} z_{n}^{2}+\frac{n-1}{n}-\frac{1}{(n-1)(n-2)} .
\end{aligned}
$$

Note that

$$
z_{n}^{2} \leq \frac{n-1}{n}
$$

where equality holds if and only if

$$
z_{1}, \cdots, z_{n-1}= \pm \frac{1}{\sqrt{n(n-1)}}
$$

Therefore we have

$$
g\left(z_{1}, \cdots, z_{n}\right) \leq(n-2)^{2}[n(n-1)]^{-1},
$$

where equality holds if and only if at least $n-1$ numbers of $z_{i}$ 's are equal. From (1.15) and (1.18)

$$
\left|\sum_{i} x_{i} y_{i}^{2}\right| \leq \max \left\{\left|M_{0}\right|,\left|m_{0}\right|\right\} \leq(n-2)[n(n-1)]^{-1 / 2},
$$


where $M_{0}$ and $m_{0}$ are the maximum and minimum of $f$ under the constraint condition (1.11) respectively. The above computation implies that the equalities in (1.19) hold if and only if at least $n-1$ pairs of the $\left(x_{i}, y_{i}\right)$ 's are equal.

COROLlary 1.7. If $a_{1}, \cdots, a_{n}$ are $n$ real numbers with $\sum_{i} a_{i}=0$, then

$$
\begin{aligned}
\left|\sum_{i} a_{i}^{3}\right| & \leq(n-2)[n(n-1)]^{-1 / 2}\left(\sum_{i} a_{i}^{2}\right)^{3 / 2}, \\
\sum_{i} a_{i}^{4} & \leq \frac{n^{2}-3 n+3}{n(n-1)}\left(\sum_{i} a_{i}^{2}\right)^{2} .
\end{aligned}
$$

Moreover the following (1), (2) and (3) are equivalent.

(1) The equality in (1.20) holds.

(2) The equality in (1.21) holds.

(3) At least $n-1$ numbers of the $a_{i}$ 's are equal.

\section{§. Geometric inequalities for the second fundamental form}

Throughout this section let $M^{n}$ be an oriented closed submanifold of dimension $n$ in an $(n+p)$-dimensional manifold $N^{n+p}$ with parallel mean curvature normal field. The squared norm of the second fundamental form is divided into $S_{H}$ and $S_{I}$, which we shall call the semi-norms of the second fundamental form. The geometric inequalities for the semi-norms are provided in this section. In Theorems 2.4 and 2.8 we need not assume the completeness of $N$.

From (1.6), (1.7) and Proposition 1.2,

$$
\Delta h_{i j}^{n+1}=-\sum_{k}\left(K_{n+1 k i k j}+K_{n+1 i j k k}\right)+\sum_{k, m}\left(h_{m k}^{n+1} R_{m i j k}+h_{i m}^{n+1} R_{m k j k}\right) .
$$

Substituting (1.3) into the above, we get

$$
\begin{aligned}
\Delta h_{i j}^{n+1} & =-\sum_{k}\left(K_{n+1 k i k j}+K_{n+1 i j k k}\right)+\sum_{m, k}\left(h_{m i}^{n+1} K_{m k j k}+h_{m k}^{n+1} K_{m i j k}\right) \\
& +\sum_{m, k, \alpha}\left(h_{m i}^{n+1} h_{m j}^{\alpha} h_{k k}^{\alpha}+h_{k m}^{n+1} h_{k i}^{\alpha} h_{m j}^{\alpha}-h_{k m}^{n+1} h_{k m}^{\alpha} h_{i j}^{\alpha}-h_{m i}^{n+1} h_{m k}^{\alpha} h_{k j}^{\alpha}\right)
\end{aligned}
$$


Therefore

$$
\frac{1}{2} \Delta S_{H}=\sum_{i, j, k}\left(h_{i j k}^{n+1}\right)^{2}+\sum_{i, j, k} h_{i j}^{n+1} \Delta h_{i j}^{n+1}=A+B+C .
$$

Here we set

$$
\begin{aligned}
A:= & n H \operatorname{tr} H_{n+1}^{3}-\left(\operatorname{tr} H_{n+1}^{2}\right)^{2}-\sum_{\beta \neq n+1}\left[\operatorname{tr}\left(H_{n+1} H_{\beta}\right)\right]^{2}, \\
B:= & \sum_{i, j, k, m}\left(h_{i j}^{n+1} h_{m j}^{n+1} K_{m k i k}+h_{i j}^{n+1} h_{m k}^{n+1} K_{m i j k}\right), \\
C:= & \sum_{i, j, k}\left(h_{i j k}^{n+1}\right)^{2}-\sum_{i, j, k}\left(h_{i j}^{n+1} K_{n+1 k i k j}+h_{i j}^{n+1} K_{n+1 i j k k}\right) \\
& +\sum_{\beta \neq n+1} \operatorname{tr}\left(H_{n+1} H_{\beta}\right)^{2}-\sum_{\beta \neq n+1} \operatorname{tr}\left(H_{n+1}^{2} H_{\beta}^{2}\right) .
\end{aligned}
$$

Several lemmas will be needed for the proof of our geometric inequalities. We first find the lower bound for $A$ in terms of $H, S_{H}$ and $S$.

LEMMA 2.1.

$$
A \geq\left(S_{H}-n H^{2}\right)\left[2 n H^{2}-S-\frac{n(n-2)}{\sqrt{n(n-1)}} H\left(S_{H}-n H^{2}\right)^{1 / 2}\right] .
$$

Proof. Let $\left\{e_{i}\right\}$ be an orthonormal frame at a point on $M$ such that the matrix $H_{n+1}$ takes the diagonal form and such that $h_{i j}^{n+1}=\lambda_{i}^{n+1} \delta_{i j}$ for all $i, j$. Set

$$
\begin{array}{r}
f_{k}:=\sum_{i}\left(\lambda_{i}^{n+1}\right)^{k}, \quad B_{k}:=\sum_{i}\left(\mu_{i}^{n+1}\right)^{k}, \quad \mu_{i}^{n+1}:=H-\lambda_{i}^{n+1}, \\
\quad \text { for } i=1, \cdots, n .
\end{array}
$$

Then

$$
B_{1}=0, \quad B_{2}=S_{H}-n H^{2}
$$

and

$$
B_{3}=3 H S_{H}-2 n H^{3}-f_{3} .
$$

From (2.2), (2.3) and Corollary 1.7, we get

$$
A=n H f_{3}-S_{H}^{2}-\sum_{\beta \neq n+1}\left[\sum_{i, j}\left(h_{i j}^{n+1}-H \delta_{i j}\right) h_{i j}^{\beta}\right]^{2}
$$




$$
\begin{aligned}
& \geq n H\left[3 H S_{H}-2 n H^{3}-\frac{n-2}{\sqrt{n(n-1)}} B_{2}^{3 / 2}\right]-S_{H}^{2}-\sum_{\beta \neq n+1}\left(\sum_{i} \mu_{i}^{n+1} h_{i i}^{\beta}\right)^{2} \\
& \geq\left(S_{H}-n H^{2}\right)\left[2 n H^{2}-\frac{n(n-2)}{\sqrt{n(n-1)}} H B_{2}^{1 / 2}\right]+n H^{2} S_{H}-S_{H}^{2}-B_{2} S_{I} \\
& =\left(S_{H}-n H^{2}\right)\left[2 n H^{2}-S-\frac{n(n-2)}{\sqrt{n(n-1)}} H\left(S_{H}-n H^{2}\right)^{1 / 2}\right] .
\end{aligned}
$$

This proves Lemma 2.1.

The lower bound of $B$ in terms of $a, H$ and $S_{H}$ is obtained as follows.

LEMMA 2.2 .

$$
B \geq n a\left(S_{H}-n H^{2}\right)
$$

Proof. It follows that

$$
\begin{aligned}
B & =\sum_{i, k}\left(\lambda_{i}^{n+1}\right)^{2} K_{i k i k}+\sum_{i, k} \lambda_{i}^{n+1} \lambda_{k}^{n+1} K_{k i i k} \\
& =\frac{1}{2} \sum_{i, k}\left(\lambda_{i}^{n+1}-\lambda_{k}^{n+1}\right)^{2} K_{i k i k} \geq \frac{1}{2} a \sum_{i, k}\left(\lambda_{i}^{n+1}-\lambda_{k}^{n+1}\right)^{2} \\
& =n a\left(S_{H}-n H^{2}\right) .
\end{aligned}
$$

This proves Lemma 2.2.

The integral of $C$ is estimated as

LEMMA 2.3 .

$$
\int_{M} C d M \geq-\frac{1}{72} n(n-1)(26 n+16 p-41) \int_{M}(b-a)^{2} d M .
$$

Proof. First of all we note that

$$
\begin{aligned}
-\sum_{i, j, k}\left(h_{i k}^{n+1} K_{n+1 j i j k}+h_{i j}^{n+1} K_{n+1 i j k k}\right) & \\
=-\sum_{i, j, k} \nabla_{k}\left(h_{i k}^{n+1} K_{n+1 j i j}+h_{i j}^{n+1} K_{n+1 i j k}\right) & \\
& +\sum_{i, j, k}\left(h_{i k k}^{n+1} K_{n+1 j i j}+h_{i j k}^{n+1} K_{n+1 i j k}\right) .
\end{aligned}
$$


We define a differentiable 1 -form $\omega$ as follows.

$$
\omega=\sum_{i, j, k}\left(h_{i k}^{n+1} K_{n+1 j i j}+h_{i j}^{n+1} K_{n+1 i j k}\right) \omega_{k} .
$$

We then get

$$
\operatorname{div} \omega=\sum_{i, j, k} \nabla_{k}\left(h_{i k}^{n+1} K_{n+1 j i j}+h_{i j}^{n+1} K_{n+1 i j k}\right) .
$$

Thus,

$$
\begin{gathered}
C=\sum_{i, j, k}\left(h_{i j k}^{n+1}\right)^{2}+\sum_{i, j, k}\left(h_{i k k}^{n+1} K_{n+1 j i j}+h_{i j k}^{n+1} K_{n+1 i j k}\right) \\
-\operatorname{div} \omega+\sum_{i, j, m, \beta \neq n+1} h_{i j}^{n+1} h_{m j}^{\beta} K_{\beta n+1 m i} .
\end{gathered}
$$

Since the mean curvature normal field of $M$ is parallel,

$$
\sum_{i} h_{i i j}^{n+1}=0, \quad \text { for all } j .
$$

From (1.6), (2.4) and Proposition 1.5,

$$
\begin{aligned}
\sum_{i, j, k} h_{i k k}^{n+1} K_{n+1 j i j} & =\sum_{i, j, k}\left(h_{k k i}^{n+1}-K_{n+1 k i k}\right) K_{n+1 j i j} \\
& \geq-\sum_{i}\left(\sum_{j} K_{n+1 j i j}\right)^{2} \\
& \geq-\frac{1}{4} n(n-1)^{2}(b-a)^{2} .
\end{aligned}
$$

On the other hand, $\sum_{i, j, k}\left(h_{i j k}^{n+1}+\frac{1}{2} K_{n+1 i j k}\right)^{2} \geq 0$ implies that

$$
\begin{aligned}
\sum_{i, j, k}\left(h_{i j k}^{n+1}\right)^{2}+\sum_{i, j, k} h_{i j k}^{n+1} & K_{n+1 i j k} \\
& \geq-\frac{1}{4} \sum_{i, j, k} K_{n+1 i j k}^{2} \\
& \geq-\frac{1}{4} \sum_{i \neq j \neq k \neq i} K_{n+1 i j k}^{2}-\frac{1}{2} \sum_{i \neq j} K_{n+1 i j i}^{2} \\
& \geq-\frac{1}{72} n(n-1)(8 n-7)(b-a)^{2} .
\end{aligned}
$$


Also from (1.4),

$$
\begin{aligned}
& \sum_{i, j, m, \beta \neq n+1} h_{i j}^{n+1} h_{m j}^{\beta} K_{\beta n+1 m i} \\
& \quad=\frac{1}{2} \sum_{i, j, m, \beta \neq n+1}\left(h_{i j}^{n+1} h_{m j}^{\beta}+h_{i j}^{\beta} h_{m j}^{n+1}\right) K_{\beta n+1 m i}-\frac{1}{2} \sum_{i, m, \beta \neq n+1} K_{\beta n+1 m i}^{2} \\
& \quad \geq-\frac{2}{9}(p-1) n(n-1)(b-a)^{2} .
\end{aligned}
$$

Here we note that $h_{i j}^{n+1} h_{m j}^{\beta}+h_{i j}^{\beta} h_{m j}^{n+1}$ is symmetric with respect to $i$ and $m$, while $K_{\beta n+1 m i}$ is anti-symmetric with respect to $i$ and $m$. Therefore

$$
\sum_{i, j, m, \beta \neq n+1}\left(h_{i j}^{n+1} h_{m j}^{\beta}+h_{i j}^{\beta} h_{m j}^{n+1}\right) K_{\beta n+1 m i}=0 .
$$

Thus we have

$$
C \geq-\frac{1}{72} n(n-1)(26 n+16 p-41)(b-a)^{2}-\operatorname{div} \omega
$$

By using Green's divergence theorem we get

$$
\int_{M} C d M \geq-\frac{1}{72} n(n-1)(26 n+16 p-41) \int_{M}(b-a)^{2} d M
$$

We are now in position to establish the following geometric inequality by setting the constant

$$
D(n, p):=\frac{1}{72} n(n-1)(26 n+16 p-41) .
$$

THEOREM 2.4. Let $a(x)$ and $b(x)$ for a point $x \in N^{n+p}$ be the minimum and maximum of the sectional curvature of $N$ at the point respectively. Then

$$
\begin{array}{r}
\int_{M}\left\{\left(S_{H}-n H^{2}\right)\left[n a+2 n H^{2}-S-\frac{n(n-2)}{\sqrt{n(n-1)}} H\left(S_{H}-n H^{2}\right)^{1 / 2}\right]\right. \\
\left.-D(n, p)(b-a)^{2}\right\} d M \leq 0 .
\end{array}
$$


Proof. It follows from Lemmas 2.1, 2.2 and (2.1) that

$$
\begin{aligned}
\frac{1}{2} \Delta S_{H} \geq\left(S_{H}-n H^{2}\right) & {\left[n a+2 n H^{2}-S\right.} \\
& \left.-\frac{n(n-2)}{\sqrt{n(n-1)}} H\left(S_{H}-n H^{2}\right)^{1 / 2}\right]+C .
\end{aligned}
$$

Integrating both sides of (2.9) and applying Lemma 2.3 , we conclude the proof.

If $p>1$, then (1.3), (1.6) and (1.7) imply that for all $\beta \neq n+1$,

$$
\begin{aligned}
\Delta h_{i j}^{\beta}= & -\sum_{k}\left(K_{\beta k i k j}+K_{\beta i j k k}\right)+\sum_{k, m}\left(h_{m k}^{\beta} K_{m i j k}+h_{i m}^{\beta} K_{m k j k}\right) \\
& +\sum_{\alpha, m, k}\left(h_{m i}^{\beta} h_{m j}^{\alpha} h_{k k}^{\alpha}+h_{m k}^{\beta} h_{k i}^{\alpha} h_{m j}^{\alpha}-h_{m i}^{\beta} h_{m k}^{\alpha} h_{k j}^{\alpha}-h_{k m}^{\beta} h_{k m}^{\alpha} h_{i j}^{\alpha}\right) \\
& +\sum_{k, \alpha \neq n+1} h_{k i}^{\alpha} K_{\alpha \beta j k}+\sum_{k, l, \alpha \neq n+1}\left(h_{j l}^{\alpha} h_{l k}^{\beta}-h_{k l}^{\alpha} h_{l j}^{\beta}\right) h_{k i}^{\alpha} .
\end{aligned}
$$

Therefore we have

$$
\begin{aligned}
\frac{1}{2} \Delta S_{I} & =\sum_{i, j, k, \beta \neq n+1}\left(h_{i j k}^{\beta}\right)^{2}+\sum_{i, j, \beta \neq n+1} h_{i j}^{\beta} \Delta h_{i j}^{\beta} \\
& =W+X+Y+Z
\end{aligned}
$$

where we set

$$
W:=-\sum_{\alpha, \beta \neq n+1} N\left(H_{\alpha} H_{\beta}-H_{\beta} H_{\alpha}\right)-\sum_{\alpha, \beta \neq n+1}\left[\operatorname{tr}\left(H_{\alpha} H_{\beta}\right)\right]^{2},
$$

$$
X:=n H \sum_{\beta \neq n+1} \operatorname{tr}\left(H_{n+1} H_{\beta}^{2}\right)-\sum_{\beta \neq n+1}\left[\operatorname{tr}\left(H_{n+1} H_{\beta}\right)\right]^{2},
$$

$$
\begin{aligned}
(2.12) \quad Y:= & \sum_{i, j, k, m, \beta \neq n+1}\left(h_{i j}^{\beta} h_{j m}^{\beta} K_{m k i k}+h_{m k}^{\beta} h_{i j}^{\beta} K_{m i j k}\right) \\
& +\sum_{i, j, k, \alpha, \beta \neq n+1} h_{i k}^{\alpha} h_{i j}^{\beta} K_{\alpha \beta j k}, \\
(2.13) \quad Z:= & \sum_{i, j, k, \beta \neq n+1}\left(h_{i j k}^{\beta}\right)^{2}-\sum_{i, j, k, \beta \neq n+1}\left(h_{i j}^{\beta} K_{\beta k i k j}+h_{i j}^{\beta} K_{\beta i j k k}\right) \\
& +\sum_{\beta \neq n+1} \operatorname{tr}\left(H_{n+1} H_{\beta}\right)^{2}-\sum_{\beta \neq n+1} \operatorname{tr}\left(H_{n+1}^{2} H_{\beta}^{2}\right) .
\end{aligned}
$$


From Proposition 1.4 we find a lower bound for the first term of right hand side of $(2.10)$ as

$$
W \geq-\left(1+\frac{1}{2} \operatorname{sgn}(p-2)\right)\left(\sum_{\beta \neq n+1} \operatorname{tr} H_{\beta}^{2}\right)^{2} .
$$

To estimate the lower bounds for the other terms on the right hand side of (2.10) we need the following Lemmas.

LEMMA 2.5.

$$
\begin{aligned}
X \geq\left[2 n H^{2}-(n-2) n^{1 / 2}(n-1)^{-1 / 2} H\left(\operatorname{tr} H_{n+1}^{2}\right.\right. & \left.-n H^{2}\right)^{1 / 2} \\
& \left.-\operatorname{tr} H_{n+1}^{2}\right] \sum_{\beta \neq n+1} \operatorname{tr} H_{\beta}^{2} .
\end{aligned}
$$

Proof. We rewrite (2.11) as

$$
\begin{aligned}
X=n H \sum_{\beta \neq n+1} \operatorname{tr}[ & \left.\left(H_{n+1}-H I\right) H_{\beta}^{2}\right] \\
& +n H^{2} \sum_{\beta \neq n+1} \operatorname{tr} H_{\beta}^{2}-\sum_{\beta \neq n+1}\left[\operatorname{tr}\left(H_{n+1}-H I\right) H_{\beta}\right]^{2},
\end{aligned}
$$

where $I$ is the unit $(n \times n)$-matrix. Fix a vector $e_{\beta}$ and let $\left\{e_{i}\right\}$ be a local orthonormal frame such that the matrix $H_{\beta}$ takes the diagonal form and such that

$$
h_{i j}^{\beta}=0, \quad \text { for } i \neq j
$$

Then we get

$$
\begin{aligned}
n H \operatorname{tr}\left[\left(H_{n+1}-\right.\right. & \left.H I) H_{\beta}^{2}\right]-\left[\operatorname{tr}\left(H_{n+1}-H I\right) H_{\beta}\right]^{2} \\
& =n H\left[\sum_{i}\left(h_{i i}^{n+1}-H\right)\left(h_{i i}^{\beta}\right)^{2}\right]-\left[\sum_{i}\left(h_{i i}^{n+1}-H\right) h_{i i}^{\beta}\right]^{2} .
\end{aligned}
$$

Using the Schwarz inequality and Proposition 1.6, we see from (2.16)

$$
\begin{aligned}
n H \operatorname{tr}[ & \left.\left(H_{n+1}-H I\right) H_{\beta}^{2}\right]-\left[\operatorname{tr}\left(H_{n+1}-H I\right) H_{\beta}\right]^{2} \\
\geq & -(n-2) n^{1 / 2}(n-1)^{-1 / 2} H\left[\sum_{i}\left(h_{i i}^{n+1}-H\right)^{2}\right]^{1 / 2}\left[\sum_{i}\left(h_{i i}^{\beta}\right)^{2}\right] \\
& -\left[\sum_{i}\left(h_{i i}^{n+1}-H\right)^{2}\right]\left[\sum_{i}\left(h_{i i}^{\beta}\right)^{2}\right] \\
\geq & -(n-2) n^{1 / 2}(n-1)^{-1 / 2} H\left(\operatorname{tr} H_{n+1}^{2}-n H^{2}\right)^{1 / 2} \operatorname{tr} H_{\beta}^{2} \\
& -\left(\operatorname{tr} H_{n+1}^{2}-n H^{2}\right) \operatorname{tr} H_{\beta}^{2} .
\end{aligned}
$$


This together with (2.15) implies the desired inequality.

LEMMA 2.6.

$$
Y \geq n a \sum_{\beta \neq n+1} \operatorname{tr} H_{\beta}^{2}-\frac{2}{3}(p-2)(n-1)^{1 / 2}(b-a) \sum_{\beta \neq n+1} \operatorname{tr} H_{\beta}^{2}
$$

Proof. For a fixed index $\beta$ we choose an orthonormal frame $\left\{e_{i}\right\}$ such that the matrix $H_{\beta}$ takes the diagonal form. We then conclude the proof by

$$
\begin{aligned}
& \sum_{i, j, k, m}\left(h_{i j}^{\beta} h_{j m}^{\beta} K_{m k i k}+h_{i j}^{\beta} h_{m k}^{\beta} K_{m i j k}\right)+\sum_{i, j, k, \alpha \neq n+1} h_{i k}^{\alpha} h_{i j}^{\beta} K_{\alpha \beta j k} \\
&=\sum_{i, k}\left(h_{i i}^{\beta}\right)^{2} K_{i k i k}+\sum_{i, k} h_{k k}^{\beta} h_{i i}^{\beta} K_{k i i k}+\sum_{i, k, \alpha \neq n+1} h_{i k}^{\alpha} \lambda_{i}^{\beta} K_{\alpha \beta i k} \\
& \geq \frac{1}{2} \sum_{i, k}\left(h_{i i}^{\beta}-h_{k k}^{\beta}\right)^{2} K_{i k i k}-\sum_{i \neq k, \alpha \neq \beta, n+1} \frac{2}{3}(b-a)\left|h_{i k}^{\alpha} \lambda_{i}^{\beta}\right| \\
& \geq \frac{1}{2} a \sum_{i, k}\left(h_{i i}^{\beta}-h_{k k}^{\beta}\right)^{2} \\
& \quad-\sum_{i \neq k, \alpha \neq \beta, n+1} \frac{1}{3}(b-a)\left[(n-1)^{1 / 2}\left(h_{i k}^{\alpha}\right)^{2}+(n-1)^{-1 / 2}\left(\lambda_{i}^{\beta}\right)^{2}\right] \\
& \geq n a \cdot \operatorname{tr} H_{\beta}^{2}-\frac{1}{3}(n-1)^{1 / 2}(b-a) \sum_{\alpha \neq \beta, n+1} \operatorname{tr} H_{\alpha}^{2} \\
& \quad-\frac{1}{3}(n-1)^{1 / 2}(p-2)(b-a) \operatorname{tr} H_{\beta}^{2} .
\end{aligned}
$$

LEMma 2.7.

$$
\int_{M} Z d M \geq-\frac{1}{72}(p-1) n(n-1)(26 n-9) \int_{M}(b-a)^{2} d M .
$$

Proof. A differentiable 1-form $\theta$ is defined as follows

$$
\theta:=\sum_{i, j, k, \beta \neq n+1}\left(h_{i k}^{\beta} K_{\beta j i j}+h_{i j}^{\beta} K_{\beta i j k}\right) \omega_{k} .
$$


We then have

$$
\begin{aligned}
-\sum_{i, j, k, \beta \neq n+1}\left(h_{i j}^{\beta} K_{\beta k i k j}+h_{i j}^{\beta} K_{\beta i j k k}\right) & \\
& =\sum_{i, j, k, \beta \neq n+1}\left(h_{i j j}^{\beta} K_{\beta k i k}+h_{i j k}^{\beta} K_{\beta i j k}\right)-\operatorname{div} \theta .
\end{aligned}
$$

From $\sum_{i, j, k}\left(h_{i j k}^{\beta}+\frac{1}{2} K_{\beta i j k}\right)^{2} \geq 0$ we have

$$
\begin{aligned}
\sum_{i, j, k, \beta \neq n+1} & {\left[\left(h_{i j k}^{\beta}\right)^{2}+h_{i j k}^{\beta} K_{\beta i j k}\right] } \\
& \geq-\frac{1}{4} \sum_{i, j, k, \beta \neq n+1} K_{\beta i j k}^{2} \\
& \geq-\frac{1}{4} \sum_{i \neq j \neq k \neq i, \beta \neq n+1} K_{\beta i j k}^{2}-\frac{1}{2} \sum_{i \neq j, \beta \neq n+1} K_{\beta i j i}^{2} \\
& \geq-\frac{1}{72}(p-1) n(n-1)(8 n-7)(b-a)^{2} .
\end{aligned}
$$

Since $M$ admits parallel mean curvature normal field, we have

$$
\sum_{i} h_{i i j}^{\beta}=0, \quad \text { for all } j
$$

From (1.6), (2.19) and Proposition 1.5 we get

$$
\begin{aligned}
\sum_{i, j, k, \beta \neq n+1} h_{i j j}^{\beta} K_{\beta k i k} & =-\sum_{i, \beta \neq n+1}\left(\sum_{j} K_{\beta j i j}\right)^{2} \\
& \geq-\frac{1}{4}(p-1) n(n-1)^{2}(b-a)^{2} .
\end{aligned}
$$

From (1.4) and Proposition 1.2,

$$
\begin{aligned}
& \sum_{\beta \neq n+1} \operatorname{tr}\left(H_{n+1} H_{\beta}\right)^{2}-\sum_{\beta \neq n+1} \operatorname{tr}\left(H_{n+1}^{2} H_{\beta}^{2}\right) \\
& \quad=\frac{1}{2} \sum_{i, j, k, \beta \neq n+1}\left(h_{i j}^{n+1} h_{i k}^{\beta}+h_{i k}^{n+1} h_{i j}^{\beta}\right) K_{n+1 \beta j k}-\frac{1}{2} \sum_{j, k, \beta \neq n+1} K_{n+1 \beta j k}^{2} \\
& \quad \geq-\frac{2}{9}(p-1) n(n-1)(b-a)^{2} .
\end{aligned}
$$


Combining (2.13), (2.17), (2.18), (2.20) and the above inequality, we get

$$
Z \geq-\frac{1}{72}(p-1) n(n-1)(26 n-9)(b-a)^{2}-\operatorname{div} \theta
$$

and hence,

$$
\int_{M} Z d M \geq-\frac{1}{72}(p-1) n(n-1)(26 n-9) \int_{M}(b-a)^{2} d M .
$$

We now set

$$
E(n, p):=\frac{2}{3}(p-2)(n-1)^{1 / 2}, \quad F(n, p):=\frac{1}{72}(p-1) n(n-1)(26 n-9) .
$$

THEOREM 2.8. If $p \neq 1$, then

$$
\begin{aligned}
& \int_{M}\left\{S _ { I } \left[n a+2 n H^{2}-S-\frac{1}{2}\right.\right. \operatorname{sgn}(p-2) S_{I} \\
&-(n-2) n^{1 / 2}(n-1)^{-1 / 2} H\left(S_{H}-n H^{2}\right)^{1 / 2} \\
&\left.\quad-E(n, p)(b-a)]-F(n, p)(b-a)^{2}\right\} d M \leq 0 .
\end{aligned}
$$

Proof. We see from (2.10), (2.14), Lemmas 2.5 and 2.6,

$$
\begin{aligned}
\frac{1}{2} \Delta S_{I} \geq S_{I} & {\left[n a+2 n H^{2}-S-\frac{1}{2} \operatorname{sgn}(p-2) S_{I}\right.} \\
& -(n-2) n^{1 / 2}(n-1)^{-1 / 2} H\left(S_{H}-n H^{2}\right)^{1 / 2} \\
& \left.-\frac{2}{3}(p-2)(n-1)^{1 / 2}(b-a)\right]+Z
\end{aligned}
$$

By the Green formula and Lemma 2.7,

$$
\begin{aligned}
\int_{M}\left\{S_{I}[n a\right. & +2 n H^{2}-S-\frac{1}{2} \operatorname{sgn}(p-2) S_{I} \\
& -(n-2) n^{1 / 2}(n-1)^{-1 / 2} H\left(S_{H}-n H^{2}\right)^{1 / 2} \\
& \left.-E(n, p)(b-a)]-F(n, p)(b-a)^{2}\right\} d M \leq 0 .
\end{aligned}
$$




\section{§3. Rigidity theorems for compact submanifolds}

The compact case for Main Theorem is discussed here. By setting

$$
\begin{aligned}
& \beta_{1}(n):=\frac{1}{6 \sqrt{2}} \sqrt{n(n-1)(26 n-25)}, \\
& \gamma_{1}(n):=n+\frac{1}{6 \sqrt{2}} \sqrt{n(n-1)(26 n-25)}
\end{aligned}
$$

we first prove the following.

THEOREM 3.1. Let $p=1$. There exists a number $\delta_{1}(n)$ with $0<$ $\delta_{1}(n)<1$ such that if the sectional curvature of $N^{n+1}$ and the squared norm of the second fundamental form of $M$ satisfy

$$
\delta_{1}(n) \leq K_{N} \leq 1, \quad n H^{2}+\beta_{1}(n)(1-c) \leq S \leq \alpha(n, H)-\gamma_{1}(n)(1-c),
$$

where $c:=\inf K_{N}$, then $N$ is isometric to $\mathbf{S}^{n+1}(1)$. Moreover $M$ is congruent to either $\mathbf{S}^{n}\left(\frac{1}{\sqrt{1+H^{2}}}\right)$ or the isoparametric hypersurface $\mathbf{S}^{n-1}\left(\frac{1}{\sqrt{1+\lambda^{2}}}\right) \times$ $\mathbf{S}^{1}\left(\frac{\lambda}{\sqrt{1+\lambda^{2}}}\right)$. Here $\lambda$ and $\alpha(n, H)$ are defined in Theorem 0.1 .

Proof. Since $c \leq a(x) \leq b(x) \leq 1,(2.8)$ implies that

$$
\begin{array}{r}
\int_{M}\left\{\left(S-n H^{2}\right)\left[n c+2 n H^{2}-S-\frac{n(n-2)}{\sqrt{n(n-1)}} H\left(S-n H^{2}\right)^{1 / 2}\right]\right. \\
\left.-D(n, p)(1-c)^{2}\right\} \leq 0 .
\end{array}
$$

Setting $\delta_{1}(n):=1-2 n(n-1)\left[\left(n^{2}-2 n+2\right)\left(\beta_{1}(n)+\gamma_{1}(n)\right)\right]^{-1}$, we have

$$
\begin{aligned}
1-c \leq 1-\delta_{1}(n) & =2 n(n-1)\left[\left(n^{2}-2 n+2\right)\left(\beta_{1}(n)+\gamma_{1}(n)\right)\right]^{-1} \\
& \leq \min _{H \geq 0}\left[\alpha(n, H)-n H^{2}\right]\left[\beta_{1}(n)+\gamma_{1}(n)\right]^{-1} \\
& \leq\left[\alpha(n, H)-n H^{2}\right]\left[\beta_{1}(n)+\gamma_{1}(n)\right]^{-1} .
\end{aligned}
$$

Thus we get

$$
n H^{2}+\beta_{1}(n)(1-c) \leq \alpha(n, H)-\gamma_{1}(n)(1-c) .
$$

From assumption

$$
n H^{2}+\beta_{1}(n)(1-c) \leq S \leq \alpha(n, H)-\gamma_{1}(n)(1-c),
$$


we see that the first term on the left hand side of (3.3) is not less than

$$
\begin{aligned}
& \beta_{1}(n)(1-c)\left[n+2 n H^{2}-\alpha(n, H)\right. \\
& \left.-\frac{n(n-2)}{\sqrt{n(n-1)}} H\left(\alpha(n, H)-n H^{2}\right)^{1 / 2}+\alpha(n, H)-S+n c-n\right] \\
& \quad=\beta_{1}(n)(1-c)[\alpha(n, H)-S+n(c-1)] \\
& \geq \beta_{1}(n)(1-c)\left[\gamma_{1}(n)(1-c)-n(1-c)\right] \\
& =D(n, p)(1-c)^{2} .
\end{aligned}
$$

Thus we have

$$
\begin{array}{r}
\left(S-n H^{2}\right)\left[n c+2 n H^{2}-S-\frac{n(n-2)}{\sqrt{n(n-1)}} H\left(S-n H^{2}\right)^{1 / 2}\right] \\
-D(n, p)(1-c)^{2} \geq 0 .
\end{array}
$$

From (3.1) and (3.3) we observe that the left hand side of (3.3) is identically zero. This together with (2.8) and $c \leq a \leq b \leq 1$ implies $a \equiv c$ and $b \equiv 1$. Since equalities hold on (2.5) and (2.6), we have

$$
\begin{aligned}
\sum_{i, j, k} h_{i k k}^{n+1} K_{n+1 j i j} & =-\frac{1}{4} n(n-1)^{2}(b-a)^{2}=-\frac{1}{4} n(n-1)^{2}(1-c)^{2} \\
0 & =h_{i k k}^{n+1}+\frac{1}{2} K_{n+1 i k k}=h_{i k k}^{n+1} .
\end{aligned}
$$

This implies that $1-c=0$. Therefore all the equalities hold in (2.5), (2.6), (3.1) and (3.3). Since the left hand side of (3.3) is identically zero and $c=1$, we see that $N$ is isometric to $\mathbf{S}^{n+1}(1)$, and

$$
S=n H^{2} \quad \text { or } \quad S=\alpha(n, H) .
$$

The above relations imply that $S \leq C(n, p, H)$. From Theorem 0.1 we see that $M$ is the small sphere $\mathbf{S}^{n}\left(\frac{1}{\sqrt{1+H^{2}}}\right)$ or the isoparametric hypersurface $\mathbf{S}^{n-1}\left(\frac{1}{\sqrt{1+\lambda^{2}}}\right) \times \mathbf{S}^{1}\left(\frac{\lambda}{\sqrt{1+\lambda^{2}}}\right)$.

The following lemma is needed for the discussion of the case of higher codimensions.

LEMMA 3.2. Let $M^{n}$ be a closed and oriented submanifold in $N^{n+p}$. If

$$
S \leq n d+\frac{n^{3}}{2(n-1)} H^{2}-\frac{n(n-2)}{\sqrt{2(n-1)}} \sqrt{n^{2} H^{4}+4(n-1) H^{2} d}-\alpha_{1}(d-c)
$$


then either $d=c$ or

$$
\int_{M}\left(S_{H}-n H^{2}\right) d M \leq \alpha_{2} \int_{M}(b-a) d M .
$$

Here $\alpha_{1}, \alpha_{2}$ are positive constants with $\alpha_{2}=D(n, p)\left(\alpha_{1}-n\right)^{-1}$ and $d=$ $\sup _{N} b$ as defined in $\S 1$.

Proof. Let

$$
\alpha(n, H, d):=n d+\frac{n^{3}}{2(n-1)} H^{2}-\frac{n(n-2)}{2(n-1)} \sqrt{n^{2} H^{4}+4(n-1) H^{2} d} .
$$

From assumption, the left hand side of (3.4) is not less than

$$
\begin{aligned}
& n d+2 n H^{2}-\alpha(n, H, d)-\frac{n(n-2)}{\sqrt{n(n-1)}} H\left(\alpha(n, H, d)-n H^{2}\right) \\
& \quad+\alpha(n, H, d)-S-\alpha_{1}(d-c)=a(n, H, d)-S-\alpha_{1}(d-c) \geq 0 .
\end{aligned}
$$

Then we see

(3.4) $n d-\alpha_{1}(d-c)+2 n H^{2}-S-\frac{n(n-2)}{\sqrt{n(n-1)}} H\left(S-n H^{2}\right)^{1 / 2} \geq 0$.

From Theorem 2.4,

$$
\begin{array}{r}
\int_{M}\left\{\left(S_{H}-n H^{2}\right)\left[n c+2 n H^{2}-S-\frac{n(n-2)}{\sqrt{n(n-1)}} H\left(S-n H^{2}\right)^{1 / 2}\right]\right. \\
-D(n, p)(d-c)(b-a)\} d M \leq 0 .
\end{array}
$$

From (3.4),

$$
n c+2 n H^{2}-S-\frac{n(n-2)}{\sqrt{n(n-1)}} H\left(S-n H^{2}\right)^{1 / 2} \geq \alpha_{2}^{-1} D(n, p)(d-c),
$$

where $\alpha_{2}=D(n, p)\left(\alpha_{1}-n\right)^{-1}$. Substituting the above inequality into (3.5) gives

$$
\int_{M}\left\{\left(S_{H}-n H^{2}\right)\left[\alpha_{2}^{-1} D(n, p)(d-c)\right]-D(n, p)(d-c)(b-a)\right\} d M \leq 0
$$

Therefore we have either $d=c$ or

$$
\int_{M}\left(S_{H}-n H^{2}\right) d M \leq \alpha_{2} \int_{M}(b-a) d M
$$


We continue the proof of our Main Theorem.

If $p=2$, then (2.8) and (2.22) imply that

$$
\begin{aligned}
& \int_{M}\left\{( S - n H ^ { 2 } ) \left[n a+2 n H^{2}-S\right.\right. \\
&\left.-(n-2) n^{1 / 2}(n-1)^{-1 / 2} H\left(S_{H}-n H^{2}\right)^{1 / 2}\right] \\
&\left.-(D(n, p)+F(n, p))(b-a)^{2}\right\} d M \leq 0 .
\end{aligned}
$$

This implies

$$
\begin{aligned}
& \int_{M}\left\{( S - n H ^ { 2 } ) \left[n a+2 n H^{2}-S\right.\right. \\
& \left.\left.\quad-(n-2) n^{1 / 2}(n-1)^{-1 / 2} H\left(S-n H^{2}\right)^{1 / 2}\right]-G(n)(b-a)^{2}\right\} d M \leq 0
\end{aligned}
$$

where we set

$$
G(n):=\frac{1}{36} n(n-1)(26 n-9) .
$$

By using the similar method as developed in the proof of Theorem 3.1, we obtain the following

THEOREM 3.3. Let $p=2$. There exists a number $\delta_{2}(n)$ with $0<$ $\delta_{2}(n)<1$ such that if $\delta_{2}(n) \leq K_{N} \leq 1$ and if

$$
n H^{2}+\beta_{2}(n)(1-c) \leq S \leq \alpha(n, H)-\gamma_{2}(n)(1-c),
$$

where $c:=\inf _{N} K_{N}$, then $N^{n+2}$ is isometric to $\mathbf{S}^{n+2}(1)$. Moreover $M$ is congruent to one of the following:

(1) $\mathbf{S}^{n}\left(\frac{1}{\sqrt{1+H^{2}}}\right)$.

(2) the isoparametric hypersurface $\mathbf{S}^{n-1}\left(\frac{1}{\sqrt{1+\lambda^{2}}}\right) \times \mathbf{S}^{1}\left(\frac{\lambda}{\sqrt{1+\lambda^{2}}}\right)$ in $\mathbf{S}^{n+1}(1)$.

(3) the Clifford torus $\mathbf{S}^{1}\left(r_{1}\right) \times \mathbf{S}^{1}\left(r_{2}\right)$ in $\mathbf{S}^{3}(r)$ with constant mean curvature $H_{0}$, where $r_{1}, r_{2}=\left[2\left(1+H^{2}\right) \pm 2 H_{0}\left(1+H^{2}\right)^{1 / 2}\right]^{-1 / 2}, r=$ $\left(1+H^{2}-H_{0}^{2}\right)^{-1 / 2}$, and $0 \leq H_{0} \leq H$.

Here the constants are given by

$$
\begin{aligned}
& \beta_{2}(n):=\frac{1}{6 \sqrt{2}} \sqrt{n(n-1)(26 n-9)} \\
& \gamma_{2}(n):=n+\frac{1}{6 \sqrt{2}} \sqrt{n(n-1)(26 n-9)} \\
& \delta_{2}(n):=1-2 n(n-1)\left[\left(n^{2}-2 n+2\right)\left(\beta_{2}(n)+\gamma_{2}(n)\right)\right]^{-1} .
\end{aligned}
$$


We now discuss the case where $p \geq 3$.

THEOREM 3.4. Let $p \geq 3$. There exists a number $\delta_{3}(n, p) \in(0,1)$ such that if $\delta_{3}(n, p) \leq K_{N} \leq 1$, and if

$$
\begin{aligned}
& n H^{2}+\beta_{3}(n, p)(1-c)+\beta_{4}(n, p)\left[\left(1+H^{2}\right) H\right]^{1 / 2}(1-c)^{1 / 4} \\
& \quad \leq S \leq C(n, p, H)-\gamma_{3}(n, p)(1-c)-\gamma_{4}(n, p)\left[\left(1+H^{2}\right) H\right]^{1 / 2}(1-c)^{1 / 4}
\end{aligned}
$$

then $N$ is isometric to $\mathbf{S}^{n+p}(1)$ and $M$ is congruent to one of the following:

(1) $\mathbf{S}^{n}\left(\frac{1}{\sqrt{1+H^{2}}}\right)$.

(2) the isoparametric hypersurface $\mathbf{S}^{n-1}\left(\frac{1}{\sqrt{1+\lambda^{2}}}\right) \times \mathbf{S}^{1}\left(\frac{\lambda}{\sqrt{1+\lambda^{2}}}\right)$ in $\mathbf{S}^{n}(1)$.

(3) the Clifford torus $\mathbf{S}^{1}\left(r_{1}\right) \times \mathbf{S}^{1}\left(r_{2}\right)$ in $\mathbf{S}^{3}(r)$ with constant mean curvature $H_{0}$, where $r_{1}, r_{2}=\left[2\left(1+H^{2}\right) \pm 2 H_{0}\left(1+H^{2}\right)^{1 / 2}\right]^{-1 / 2}, r=$ $\left(1+H^{2}-H_{0}^{2}\right)^{-1 / 2}$, and $0 \leq H_{0} \leq H$.

(4) the Veronese surface in $\mathbf{S}^{4}\left(\frac{1}{\sqrt{1+H^{2}}}\right)$.

Here the constants $\beta_{3}, \beta_{4}, \gamma_{3}, \gamma_{4}$ and $\delta_{3}$ are given later in the remark.

Proof. We argue the proof by deriving a contradiction. Suppose that $c \neq 1$. We then have from $(2.22)$

$$
\begin{aligned}
\int_{M}\left\{S _ { I } \left[n a+\frac{5}{2} n H^{2}-\right.\right. & \frac{3}{2} S-(n-2) n^{1 / 2}(n-1)^{-1 / 2} H\left(S_{H}-n H^{2}\right)^{1 / 2} \\
& \left.-E(n, p)(1-c)]-F(n, p)(1-c)^{2}\right\} d M \leq 0
\end{aligned}
$$

In fact, since $S=S_{H}+S_{I} \geq n H^{2}+S_{I}$ we observe

$$
\frac{5}{2} n H^{2}-\frac{3}{2} S \leq 2 n H^{2}-S-\frac{1}{2} S_{I}
$$

This together with $0 \leq b-a \leq 1-c$ implies that the left hand side of (3.8) is not greater than the left hand side of (2.22) which is nonpositive.

Note that the assumption implies

$$
S \leq C(n, p, H)-\gamma_{3}(n, p)(1-c) \leq \alpha(n, H)-\alpha_{1}(n, p)(1-c)
$$


This shows that the assumption of Lemma 3.2 is fulfilled, where $d=1$. By the Schwarz inequality and Lemma 3.2,

$$
\begin{aligned}
\int_{M} H S_{I}\left(S_{H}-\right. & \left.n H^{2}\right)^{1 / 2} d M \\
& \leq H \max S_{I} \cdot \operatorname{vol}(M)^{1 / 2}\left[\int_{M}\left(S_{H}-n H^{2}\right)\right]^{1 / 2} \\
& \leq \frac{2}{3} n \alpha_{2}(n, p)^{1 / 2} H\left(1+H^{2}\right)(1-c)^{1 / 2} \operatorname{vol}(M) .
\end{aligned}
$$

Here the second inequality is obtained as follows. By Lemma 3.2,

$$
\int_{M}\left(S_{H}-n H^{2}\right) d M \leq \alpha_{2}(1-c) \operatorname{vol}(M) .
$$

This together with $S_{I} \leq S-n H^{2} \leq C(n, p, H)-n H^{2} \leq \frac{2}{3} n\left(1+H^{2}\right)$ implies

$$
\begin{aligned}
H \max S_{I} \operatorname{vol}(M)^{1 / 2}\left[\int _ { M } \left(S_{H}\right.\right. & \left.\left.-n H^{2}\right) d M\right]^{1 / 2} \\
\leq & \frac{2}{3} n \alpha_{2}(n, p)^{1 / 2} H\left(1+H^{2}\right)(1-c)^{1 / 2} \operatorname{vol}(M)
\end{aligned}
$$

Combining (3.8) and (3.9), we have

$$
\begin{aligned}
\int_{M}\left\{S_{I}[n c\right. & \left.+\frac{5}{2} n H^{2}-\frac{3}{2} S-E(n, p)(1-c)\right] \\
& \left.-E_{1}(n, p, H)(1-c)^{1 / 2}-F(n, p)(1-c)^{2}\right\} d M \leq 0
\end{aligned}
$$

where we set

$$
\begin{aligned}
& E_{1}(n, p, H) \\
& \quad:=\frac{2}{3} n^{3 / 2}(n-2)(n-1)^{-1 / 2} D(n, p)^{1 / 2}\left(\alpha_{1}(n, p)-n\right)^{-1 / 2}\left(1+H^{2}\right) H .
\end{aligned}
$$

Since $C(n, p, H) \leq \frac{1}{3}\left(2 n+5 n H^{2}\right)$ and $\gamma_{3}(n, p) \geq \frac{2}{3}\left[n+E(n, p)+F(n, p)^{1 / 2}\right\rceil$ and $\gamma_{4}(n, p)\left[\left(1+H^{2}\right) H\right]^{1 / 2}=\frac{2}{3} E_{1}(n, p, H)^{1 / 2}$, we obtain

$$
\begin{aligned}
S \leq \frac{1}{3}\left(2 n+5 n H^{2}\right)-\frac{2}{3}\left(n+E(n, p)+F(n, p)^{1 / 2}\right)(1-c) & \\
& -\frac{2}{3} E_{1}(n, p, H)^{1 / 2}(1-c)^{1 / 4} .
\end{aligned}
$$


Substituting the above into (3.10), we get

$$
\int_{M} S_{I} d M \leq\left[F(n, p)^{1 / 2}(1-c)+E_{1}(n, p, H)^{1 / 2}(1-c)^{1 / 4}\right] \operatorname{vol}(M)
$$

and this together with Lemma 3.2 yields

$$
\begin{aligned}
\int_{M}\left(S-n H^{2}\right) d M \leq & {\left[\left(\alpha_{2}(n, p)+F(n, p)^{1 / 2}\right)(1-c)\right.} \\
+ & \left.E_{1}(n, p, H)^{1 / 2}(1-c)^{1 / 4}\right] \operatorname{vol}(M) .
\end{aligned}
$$

From assumption follows

$$
S-n H^{2} \geq \beta_{3}(n, p)(1-c)+\beta_{4}(n, p)\left[\left(1+H^{2}\right) H\right]^{1 / 2}(1-c)^{1 / 4} .
$$

This and (3.12) imply that

$$
S-n H^{2} \equiv\left[\alpha_{2}(n, p)+F(n, p)^{1 / 2}\right](1-c)+E_{1}(n, p, H)^{1 / 2}(1-c)^{1 / 4} .
$$

Therefore we see that all the inequalities in (2.18), (2.20), (2.22), (3.8) and (3.12) are actually equalities. From (2.22), (3.8) and (3.12) we get $1-c=b-a$. Because $c \leq a \leq b \leq 1$, we have $a=c, b=1$. It follows from (2.18) and (2.20) that

$$
0=h_{i j j}^{\beta}+\frac{1}{2} K_{\beta i j j}=h_{i j j}^{\beta}, \quad \sum h_{i j j}^{\beta} K_{\beta k i k}=-\frac{1}{4}(p-1) n(n-1)^{2}(1-c)^{2} .
$$

This implies $1-c=0$, contradicting to what is supposed at the beginning of the proof. Since $N$ is assumed to be complete and simply connected, $N$ is isometric to $\mathbf{S}^{n+p}(1)$. Moreover from (3.5) and (3.10),

$$
S=n H^{2} \quad \text { or } \quad S=C(n, p, H) .
$$

From Theorem 0.1 we see that $M$ is one of the (1), (2), (3) and (4). This proves Theorem 3.4.

Remark 3.5. To make sure of the assumption in Theorem 3.4 we set

$$
\delta_{3}(n, p)= \begin{cases}1-\frac{4}{3}\left(\beta_{3}+\gamma_{3}\right)^{-1}, & \text { for } n=2 \\ 1-\min \left\{16\left(\beta_{3}+\gamma_{3}+\frac{5}{4} \gamma_{4}\right)^{-4},\right. & \\ \left.\frac{256}{14641}\left[n^{-1}(n-1)\left(\beta_{4}+\gamma_{4}\right)\right]^{-4}\right\}, & \text { for } n \geq 3 .\end{cases}
$$


Then the following inequality makes sense.

$$
\begin{aligned}
n H^{2}+ & \beta_{3}(n, p)(1-c)+\beta_{4}(n, p)\left[\left(1+H^{2}\right) H\right]^{1 / 2}(1-c)^{1 / 4} \\
& <C(n, p, H)-\gamma_{3}(n, p)(1-c)-\gamma_{4}(n, p)\left[\left(1+H^{2}\right) H\right]^{1 / 2}(1-c)^{1 / 4} .
\end{aligned}
$$

Here $\alpha_{1}, \alpha_{2}, \beta_{3}, \beta_{4}, \gamma_{3}$ and $\gamma_{4}$ are constants precisely given as follows.

$$
\begin{aligned}
& \alpha_{1}(n, p)=n+\frac{1}{6 \sqrt{2}}[n(n-1)(26 n+16 p-41)]^{1 / 2}, \\
& \alpha_{2}(n, p)=\frac{1}{6 \sqrt{2}}[n(n-1)(26 n+16 p-41)]^{1 / 2}, \\
& \beta_{3}(n, p)=\alpha_{2}(n, p)+F(n, p)^{1 / 2}, \\
& \gamma_{3}(n, p)=\max \left\{\alpha_{1}(n, p), \frac{2}{3}\left(n+E(n, p)+F(n, p)^{1 / 2}\right)\right\}, \\
& \beta_{4}(n, p)=3^{-3 / 4}\left[2 n^{7}(n-2)^{4}(n-1)^{-1}(26 n+16 p-41)\right]^{1 / 8}, \\
& \gamma_{4}(n, p)=3^{-7 / 4}\left[512 n^{7}(n-2)^{4}(n-1)^{-1}(26 n+16 p-41)\right]^{1 / 8} .
\end{aligned}
$$

The constants in Main Theorem are given as follows.

$$
\begin{gathered}
\tau(n, p)= \begin{cases}\delta(n, p), & \text { for } H=0 \\
\delta_{1}(n), & \text { for } p=1 \text { and } H \neq 0 \\
\delta_{2}(n), & \text { for } p=2 \text { and } H \neq 0 \\
\delta_{3}(n, p), & \text { for } p \geq 3 \text { and } H \neq 0,\end{cases} \\
A_{1}(n, p)= \begin{cases}\beta(n, p), & \text { for } H=0 \\
\beta_{1}(n), & \text { for } p=1 \text { and } H \neq 0 \\
\beta_{2}(n), & \text { for } p=2 \text { and } H \neq 0 \\
\beta_{3}(n, p), & \text { for } p \geq 3 \text { and } H \neq 0,\end{cases} \\
B_{1}(n, p)= \begin{cases}\gamma(n, p), & \text { for } H=0 \\
\gamma_{1}(n), & \text { for } p=1 \text { and } H \neq 0 \\
\gamma_{2}(n), & \text { for } p=2 \text { and } H \neq 0 \\
\gamma_{3}(n, p), & \text { for } p \geq 3 \text { and } H \neq 0,\end{cases} \\
A_{2}(n, p)= \begin{cases}\beta_{4}(n, p), & \text { for } n \geq 3, p \geq 3 \text { and } H \neq 0 \\
0, & \text { otherwise, }\end{cases} \\
B_{2}(n, p)= \begin{cases}\gamma_{4}(n, p), & \text { for } n \geq 3, p \geq 3 \text { and } H \neq 0 \\
0, & \text { otherwise. }\end{cases}
\end{gathered}
$$

Combining Theorems 0.2, 3.1, 3.3 and 3.4, we conclude the proof of Main Theorem for compact case. 


\section{$\S 4$. The complete case}

The generalized maximum principle due to Omori $[\mathrm{O}]$ and $\mathrm{Yau}[\mathrm{Y} 2]$ is the useful tool to generalize rigidity theorems such as the Chern-do CarmoKobayashi theorem to complete cases where compactness is not assumed (see $[\mathrm{H}],[\mathrm{P}])$. However it does not apply to our case because the divergence of the two 1-forms in (2.7) and (2.21) require pointwise estimates. We shall employ the following useful Lemma 4.1 for the discussion of the rigidity of complete submanifolds with parallel mean curvature normal fields where compactness is not assumed. We emphasize that all the results obtained here include the minimal case.

Lemma 4.1. (see [X-4]) Let $M^{n}$ be an n-dimensional submanifold in an $(n+p)$-dimensional Riemannian manifold $N^{n+p}$ with $K_{N} \geq c$. Here $c$ is a constant satisfying $c+H^{2}>0$. If $S \leq \alpha(n, H, c)$, then the Ricci curvature satisfies

$$
\operatorname{Ric}_{M} \geq \frac{n-1}{n}\left[n c+2 n H^{2}-S-\frac{n(n-2)}{\sqrt{n(n-1)}} H\left(S-n H^{2}\right)^{\frac{1}{2}}\right] .
$$

Moreover, if $\sup _{M}(S-\alpha(n, H, c))<0$, then $M$ is compact, where

$$
\alpha(n, H, c):=n c+\frac{n^{3}}{2(n-1)} H^{2}-\frac{n(n-2)}{2(n-1)} \sqrt{n^{2} H^{4}+4(n-1) c H^{2}} .
$$

Thus from the rigidity theorems in previous section and Lemma 4.1, we obtain the following

THEOREM 4.2. For given positive integers $n \geq 2, p$ and a nonnegative constant $H$ there exists a number $\tau(n, p)$ with $0<\tau(n, p)<1$ such that if $M^{n}$ is an oriented complete submanifold in a complete simply connected Riemannian $(n+p)$-manifold $N^{n+p}$ with $\tau(n, p) \leq K_{N} \leq 1$, and if

$$
\begin{aligned}
& n H^{2}+A_{1}(n, p)(1-c)+A_{2}(n, p)\left[\left(1+H^{2}\right) H\right]^{1 / 2}(1-c)^{1 / 4} \\
& \quad \leq S \leq C(n, p, H)-B_{1}(n, p)(1-c)-B_{2}(n, p)\left[\left(1+H^{2}\right) H\right]^{1 / 2}(1-c)^{1 / 4},
\end{aligned}
$$

then $N$ is isometric to $\mathbf{S}^{n+p}(1)$. Moreover if

$$
\sup _{M} S<\alpha(n, H)
$$

then $M$ is congruent to either $\mathbf{S}^{n}\left(\frac{1}{\sqrt{1+H^{2}}}\right)$ or the Veronese surface in $\mathbf{S}^{4}\left(\frac{1}{\sqrt{1+H^{2}}}\right)$. 
We conclude the proof of Main Theorem by the combination of Theorems 3.1, 3.3, 3.4 and 4.2.

In due course of the proof of Main Theorem the completeness and simple connectedness of $N$ is used only to guarantee that $N$ is isometric to the round sphere. We can derive $K_{N} \equiv 1$ without these assumptions. Thus we obtain the following Theorem 4.3 by using the orientable double covering.

THEOREM 4.3. For given positive integers $n \geq 2, p$ and a nonnegative constant $H$ there exists a number $\tau(n, p)$ with $0<\tau(n, p)<1$ such that if $M^{n}$ is a complete submanifold with parallel mean curvature normal field in a Riemannian $(n+p)$-manifold $N^{n+p}$ with $\tau(n, p) \leq K_{N} \leq 1$, and if

$$
\begin{aligned}
& n H^{2}+A_{1}(n, p)(1-c)+A_{2}(n, p)\left[\left(1+H^{2}\right) H\right]^{1 / 2}(1-c)^{1 / 4} \\
& \quad \leq S \leq C(n, p, H)-B_{1}(n, p)(1-c)-B_{2}(n, p)\left[\left(1+H^{2}\right) H\right]^{1 / 2}(1-c)^{1 / 4},
\end{aligned}
$$

then $K_{N} \equiv 1$. Moreover,

(1) if $\sup _{M} S<\alpha(n, H)$, then $S=n H^{2}$ and $M$ is totally umbilic, or $S=\frac{2}{3}\left(2+5 H^{2}\right)$

(2) if $M$ is compact, then $S=n H^{2}$ and $M$ is totally umbilic, or $S=$ $C(n, p, H)$.

\section{REFERENCES}

[AdC] H. Alencar and M. do Carmo, Hypersurfaces with constant mean curvature in spheres, Proc. A. M. S., 120 (1994), 1223-1229.

[CDK] S. S. Chern, M. do Carmo and S. Kobayashi, Minimal submanifolds of a sphere with second fundamental form of constant length, in Functional Analysis and Related Fields (F. E. Browder, ed.), Springer-Verlag, New York (1970).

[Ga] H. Gauchman, Minimal submanifolds of a sphere with bounded second fundamental form, Trans. A. M. S., 298 (1986), 779-791.

[G] S. Goldberg, Curvature and Homology, Academic Press, London, 1962.

[H] T. Hasanis, Characterization of totally umbilical hypersurfaces, Proc. Amer. Math. Soc., 81 (1981), 447-450.

[L1] B. Lawson, Local rigidity theorems for minimal hypersurfaces, Ann. of Math., 89 (1969), 187-197.

[L2] Rigidity theorems in rank one symmetric space, J. Differential Geometry, 4 (1970), 349-357.

[LL] A. M. Li and J. M. Li, An intrinsic rigidity theorem for minimal submanifolds in a sphere, Archiv der Math., 58 (1992), 582-594. 
[O] H. Omori, Isometric immersion of Riemannian manifold, J. Math. Soc. Japan, 19 (1967), 205-214.

[P] Y. L. Pan, Pinching theorems of Simon's type for complete minimal submanifolds in the sphere, Proc. Amer. Math. Soc., 93 (1985), 710-712.

[Sh] Y. B. Shen, On intrinsic rigidity for minimal submanifolds in a sphere, Sci. Sinica, Ser. A, 32 (1989), 769-781.

[Si] J. Simons, Minimal varieties in Riemannian manifolds, Ann. of Math., 88, (2) (1968), 62-105.

[X1] H. W. Xu, A rigidity theorem for submanifolds with parallel mean curvature in a sphere, Archiv der Math., 61 (1993), 489-496.

[X2] — On closed minimal submanifolds in pinched Riemannian manifolds, Trans. A. M. S., 347 (1995), 1743-1751.

[X3] - A pinching constant of Simons' type and isometric immersion, Chinese Ann. Math., Ser. A, 12 (1991), 261-269.

[X4] L Lower bounds for eigenvalues of submanifolds in a Riemannian manifold (1992), no. Research Report, Zhejiang University.

[Y1] S. T. Yau, Submanifolds with constant mean curvature I, II, Amer. J. Math., 96, 97 (1974, 1975), 346-366, 76-100.

[Y2] - Harmonic functions on complete Riemannian manifolds, Comm. Pure and Appl. Math., 28 (1975), 201-228.

Katsuhiro Shiohama

Department of Mathematics, Faculty of Science and Engineering

Saga University

Saga, 840-8502, Japan

Hongwei Xu

Institute of mathematics

Zhejiang University

Hangzhou, 310027-China 